\title{
Nanometric Resolved Cathodoluminescence on Few-Layer h-BN Flakes
}

\author{
A. Zobelli ${ }^{1,}$ R. Bourrellier, ${ }^{1}$ M. Amato, ${ }^{1,2}$ S. Meuret, ${ }^{1}$ L.H.G. Tizei, ${ }^{1}$ C. Giorgetti, ${ }^{2}$ A. Gloter, ${ }^{1}$ M.I. \\ Heggie, ${ }^{3}$ K. March, ${ }^{1}$ O. Stephan, ${ }^{1}$ L. Reining, ${ }^{2}$ and M. Kociak, ${ }^{1}$ \\ ${ }^{1 .}$ Laboratoire de Physique des Solides, Univ. Paris-Sud, CNRS UMR 8502, F-91405, Orsay, France \\ ${ }^{2}$ Laboratoire des Solides Irradies, Ecole Polytechnique, Route de Saclay, F-91128 Palaiseau and \\ European Theoretical Spectroscopy Facility (ETSF), France \\ ${ }^{3 .}$ Department of Chemistry, University of Surrey, Guildford GU2 7XH, United Kingdom
}

Hexagonal boron nitride (h-BN) is one of the most promising candidates for light emitting devices in the far UV region, presenting a single strong excitonic emission at $5.8 \mathrm{eV}$. However, a single line appears only in extremely pure monocrystals that can hardly be obtained only though complex synthesis processes. Common h-BN samples present more complex emission spectra that have been generally attributed to the presence of structural defects. Despite a large number of experimental studies up to now it was not possible to attribute specific emission features to well identify defective structures. Here we address this fundamental questions by adopting a theoretical and experimental approach combining few nanometer resolved cathodoluminescence techniques with high resolution transmission electron microscopy images and state of the art quantum mechanical simulations.

Very recently, we have developed a cathodoluminescence detection system integrated within a scanning transmission electron microscope. This unique experimental set up is now able to provide full emission spectra with a resolution as low as few tens of $\mathrm{meV}$ associated with an electron probe size of one nanometer. A cathodoluminescence spectrum-image can thus be recorded in parallel with an HAADF image.

Nanometric resolved cathodoluminescence on few-layer chemically exfoliated h-BN crystals have shown that emission spectra are strongly inhomogeneus within individual flakes. Emission peaks close to the free exciton appear in extended regions. Complementary investigations through high resolution transmission electron microscopy allow to associate these emission lines with extended crystal deformation such as stacking faults and folds of the planes.

By means of ab-initio calculations in the framework of Many Body Perturbation Theory (GW approximation and Bethe-Salpeter equation) we provide an in-depth description of the electronic structure and spectroscopic response of bulk hexagonal boron nitride in the presence of extended morphological modifications. In particular we show that, in a good agreement with the experimental results, additional excitons can be lighten up by symmetry distortions induced by crystal stacking faults.

Additional features appearing within the band gap present a high spatial localization, typically less than $100 \mathrm{~nm}$, and thus they can be related to individual point defects. When addressed individually through a highly focused electron probe they might exhibit a single photon emitter quantum character. This hypothesis has been recently confirmed by experiments combining our cathodoluminescence system with an Hanbury Brown and Twiss (HBT) interferometer.

\section{References}

[1] K. Watanabe et al, Nat. Mater. 3, 404 (2004)

[2] L. Zagonel et al Nano Lett. 11, 56 (2011) 
[3] R Bourrellier et al, arXiv:cond-mat/1401.1948 (2014)

a
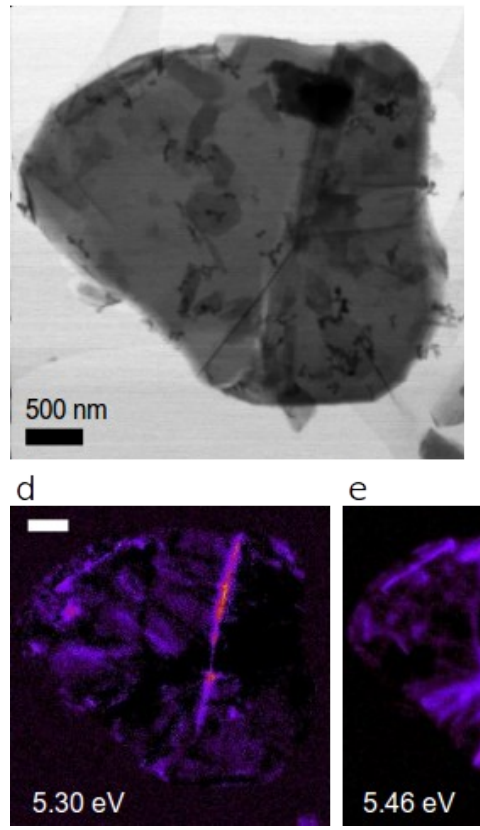

e b
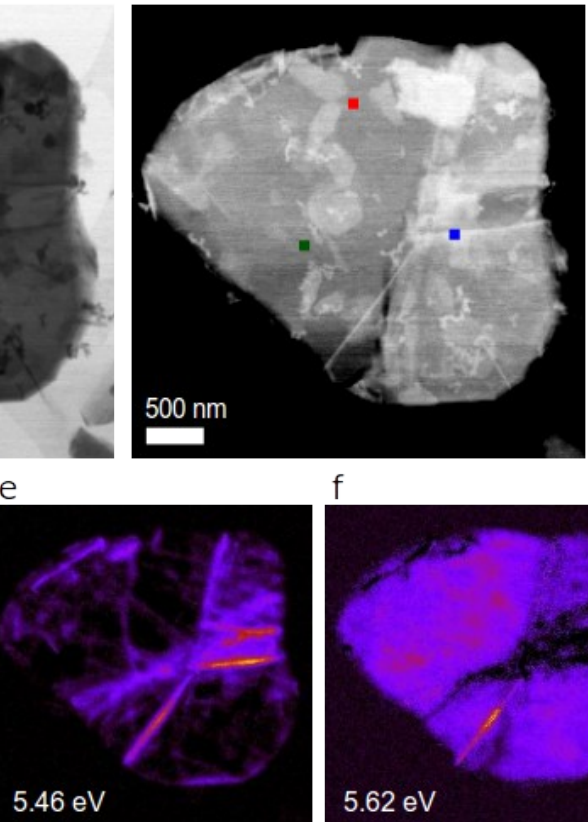

C

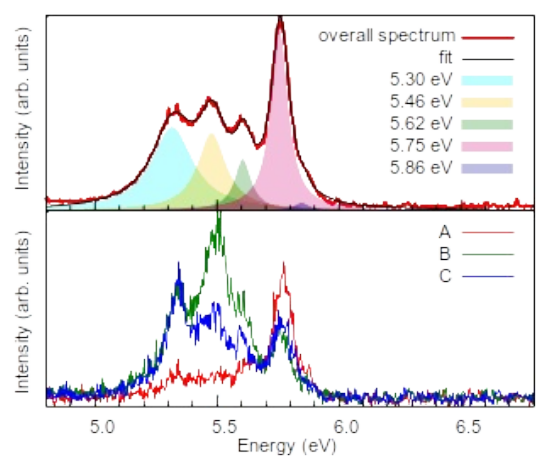

h

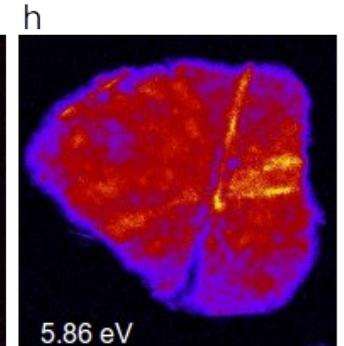

